\title{
How do women of Open Source Software support each other?
}

\author{
Vandana Singh \\ University of Tennessee, Knoxville \\ vandana@utk.edu
}

\author{
William Brandon \\ University of Tennessee, Knoxville \\ wbrando1@utk.edu
}

\begin{abstract}
This paper presents an analysis of 10,698 messages from five online forums with 1,344 participants to identify patterns of activity, major topics of discussion, and the type of social support available for participants in these Open Source Software (OSS) forums. We found that these forums serve as safe spaces shared by marginalized populations, for collaborating, networking and most importantly providing social support to each other.
\end{abstract}

\section{Introduction}

According to several estimates, in the last decade women have constituted no more than five percent of OSS communities. Some estimates indicate these numbers to be as low as one to two percent. [24, OSS4W.org] If we consider the philosophy of open source, it is disheartening to see that women do not enjoy the openness and the benefits of the movement. If these communities are open to everyone, then why do women face challenging circumstances when they participate? There are many reasons for the stagnant grown rate for women's engagement in OSS. Previous research shows us the pipeline problem, where fewer women study in technology-related fields and therefore fewer women are available for hiring in technical positions. [3, 7] The crisis is further deepened by an even smaller number of women retained in technical positions [24, 3]. The underrepresentation of women in STEM fields is well documented and, in the last two decades, we have seen a variety of approaches and initiatives to address this concern. Unfortunately, despite these efforts, we continue to observe that women's participation in STEM fields and especially in technology is nowhere equal to that of their male counterparts. Women make up almost fifty percent of the overall workforce; however, in technology, we see that this number has not increased above thirty percent [17]. Additionally, the thirty-percent number that is cited includes any woman who works in a technology field and is not an accurate representation of women employed in technical positions. Within OSS communities, we find that the numbers are even more discouraging.

If STEM fields in general, and OSS communities more specifically, are to get serious about improving gender disparity and ingrained marginalization of specific populations, they must find ways to be more inclusive beyond lip-service. In this study we explore if communities created for supporting women can be a useful approach in creating better environment for women to participate, and investigate how women engage in these communities. In our review, we have not come across another study that does so across multiple forums with the focus of this study.

At the onset, we explicate the use of the word women in the context of this study. Even though the data collected in this study is from OSS discussion forums that are named as "women of NameofSoftware", that naming is not exclusionary in nature. The term women is being used with an understanding of the monolithic nature of this term and hence the contextual definition here includes additional dimensions of gender including Trans and others outside the binary of gender identity/expression. This definition is deliberate and cognizant of the implications of intersectionality and should not be viewed as isolated to female gender. Approaching this study with this definition allows us to delve deeper into understanding the underlying issues of racism, sexism, xenophobia that perpetuate marginalization and discrimination of people in STEM communities. The forums also encourage all marginalized or "-other" participants to join the discussion.

\section{Literature Review}

Numerous scholars have addressed both the reasons for the underrepresentation of women in online communities such as OSS and what can be done to improve the situation. Ethnographic research conducted in OSS [8] has highlighted how women receive harsh treatment in OSS communities, and 
concluded that OSS is not so open for women. Many women in OSS experience "constant and extreme" sexist behavior. Furthermore, [8] explores how the concept of 'openness' is constructed in OSS communities in such a way to exclude many women from important positions and activities. Beliefs about the role gender plays in coding (e.g. a supposed "gender-blindness"), liberal individualistic attitudes, norms governing communication and how these function in the legitimization of specific coding achievements, and other factors taken together lead, the authors argue, to a situation in which women are excluded, protests notwithstanding.

It is important to address these issues in the information technology industry and, more specifically, in OSS because of the disadvantages of this type of exclusionary behavior. On one hand, we are concerned about filling information technology jobs with qualified candidates, while, on the other hand, almost half of our working population is being discouraged from joining and flourishing in this area. Including women in these positions will strengthen the global economy. The growing stereotype of IT as a male-dominated work environment needs to be rectified, and efforts need to be made to increase diversity and inclusion in these environments. The presence of diversity in software development leads to better and innovative software [8] and the current marginalization of women and other minorities is a serious deterrent to equality. Additionally, since women form half of the population and are big users of technology, it only makes sense to include them in the process of creating technology. Diversity and inclusion leads to product development that is responsive to the needs of diverse groups and hence is better for users. Very rarely, we see computing systems that are created and designed by the minority for the minority. One such example can be found in the Archives of Our Own (AO3) community, a fan fiction archive with nearly 750,000 users and over 2 million individual works [2]. AO3 was designed and coded primarily by women to meet the needs of the online fandom community. Their design decisions were informed by existing values and norms concerning issues such as accessibility, inclusivity, and identity [2], and the success of this community is evidence of the impact such efforts can have.

For women, the motivations to join OSS communities are similar to the motivations of men who join these communities. Joining and contributing to OSS projects helps in learning new programming and people/software management skills. An active presence in OSS communities leads to networking, connections and job opportunities. Participating in these communities leads to documented work experience that can be utilized for job applications and interviews. Of course, in addition, women are also motivated to contribute to OSS because it allows them to contribute to a common good and help others. Joining these projects leads to growth in knowledge and experience. Past research has advocated building a support infrastructure for women, where mentors, allies, and other women support women. In OSS online communities, women-centered online discussion forums have existed for over two decades but have not been the focus of research until recently.

A review of the literature reveals that women in technology are confronted with the dilemma of either masculinizing themselves in order to integrate into the masculine workforce or challenging the cultural system and attempting to feminize the workforce [1, 13]. [16] found that, along with other factors, access to social networks including mentors and advisors contributes to the gender imbalance among successful high-tech entrepreneurs.

Other research found that more than half of [2] women had witnessed harassment and discrimination in OSS communities. This type of unwelcoming behavior can be in the form of jokes, snide remarks and other isolating mannerisms such as ignoring posts and contributions from women or bringing unwanted attention to gender, thereby creating an uncomfortable environment. All the women respondents to their survey wrote that confidence is a critical factor, for they are entering a male-dominated field and "opening themselves to the discrimination and harassment that exists, in addition to subjecting their ideas and suggestions to the public for critiquing."

Researchers [3] discuss how the tools (e.g. software) used in OSS communities can lead to gender disparity and also discuss methods to improve the situation. The authors in [7] show that, on Stack Overflow, the presence of other women on threads influenced women's participation in those threads. More specifically, the authors found that, after posting an initial question, women on threads with other women posted again sooner than those who found themselves on threads on which there were no other women. They thus argue that 'peer parity,' or having peers with whom one can identify in communities of interaction, is crucial for women's participation in online communities and suggest the implications of this finding for mentoring and other related programs.

The concept of safe spaces for women is not a new one, and for many decades feminist literature has defined, discussed and evaluated these spaces $[14,5]$. Safe spaces emerged as physical spaces in the 1960s and were focused around creating infrastructure for women to meet, organize and be free from fear, risks and any emotional harm. [5]. Pain [21] asserts that 
space itself can become gendered because of the fear that women experience in their lives based on their perceptions of risk and the actual risks they face. These in turn have implications for the behavior of women and impacts their ability for equal participation in society. [21]. Online spaces were initially seen as a great equalizer that would provide opportunities for democratic, public, civic engagement, allowing everyone to participate equally. In reality, we have seen that the marginalized groups that were excluded in offline life face a similar discrimination in online spaces. Online abuse of women, LGBTQ and other marginalized groups is equally prevalent and vicious if not more in an online context [15, 20 and 24]. Online misogynistic abuse is a significant and troubling form of gendered violence that poses serious challenges in terms of victim-support, policy and criminal justice responses. In this research we build upon the idea of space as an infrastructure embedded in the cultural and social meaning $[14,18]$ which has implications for women's participation [21] in the male dominated field of computing. We define safe spaces as online spaces created with an objective to offer safety to women from emotional, verbal and physical harm. In this context, emotional harm includes the identity-based discrimination and harassment that leads women to leave the computing industry. Being left out then has implications for their professional and financial wellbeing.

In this research we do not suggest that every women-centered space is always experienced as safe, but the intention here is to highlight the social support that the participants in these spaces experience, as revealed by their interactions. In 1976, Cobb [22] defined social support as the information from others that one is cared for, loved, esteemed and is a part of a mutually supportive network. Social support can be studied with either a structural approach (social network, social ties, etc.) or a functional approach (functions served by social relationships in a social network) [11]. In this study we take a functional approach to understand the social support in OSS forums. Functional social support can be divided into emotional support (expressions of comfort and caring), information support (provision of advice and guidance), tangible support (provision of material aid) and belonging support (shared social activities, sense of social belonging [6]. Social support can also be differentiated into received support and perceived support. [4]. Perceived support is the perception that others will be able to provide social support and received support refers to the actual support provided by others. In this study we focus on the received social support and categorize it by the types of functional support. We also develop categories for the root messages that receive specific supportive responses, and in doing so we create a typology of the types of social support that is received and the type of social support that is sought by the women participating in these OSS forums.

\section{Research Methods 3.1 Research Questions}

The main research objective for this study is to investigate how women of open source software support each other. To achieve this objective we studied the activity level, topics of conversation, and types of support in these forums and answered the following specific research questions.

1. How active are these forums?

a) How long have the forums existed?

b) How many messages are posted on the forums?

c) How many participants post messages on the forums?

2. What are the topics of conversation on the forums?

a) Which topics of conversation occur most often on the forums?

b) What topics generate the most discussion?

3. What types of social support are being requested in these forums?

4. What types of social support do women recieve on the forums?

\subsection{Data Collection and Analysis}

3.2.1 Data Collection. To answer the above research questions, we collected data from mailing list archives from five different women-centered mailing lists. There are many types of digital forums that serve as a discussion place for women participating in OSS, including mailing lists, twitter, Facebook, chat rooms, blogs, etc. [14]. We chose mailing lists for this study because mailing lists are the most common communication channel for women of OSS, along with Internet Relay Chats (IRCs). [14]. Despite access to the IRC logs, we did not analyze those because in the discussion boards IRC was often referred to as the more private and safe space, and the authors of this research do not want to infringe on that space. Discussion forums are public and their intent is open to public as well. The women who participate in these forums are aware of the public nature of these forums.

The five mailing list archives are from GNOME, Fedora, KDE, UBUNTU, and Debian.

1. https://mail.gnome.org/archives/gnome-women$\underline{\text { list/ }}$ 
2. https://lists.fedoraproject.org/archives/list/women @lists.fedoraproject.org/

3. https://mail.kde.org/pipermail/kde-women/

4. https://lists.ubuntu.com/archives/ubuntu-women/

5. https://lists.debian.org/debian-women/

We collected all the messages in the archives for these five forums. From each of the archives, we collected the following: the message, the subject of the message, the author of the message and the date of the message. We used a spreadsheet to store all this data, and then we used NVivo software for the qualitative coding of all the messages. Our complete dataset consists of 10,698 messages from five archives spanning a range of twelve to fifteen years, posted by 1, 344 participants.

3.2.2 Data Analysis. For research question 1, we aggregated the information about each forum, the number of messages in each forum, and the number of women participating in each forum. For answering research question 2, the unit of analysis was one message. As a first step, we selected a dataset of 50 messages to develop a list of initial codes. Two independent coders reviewed these codes together to negotiate a common definition for each code category. This step resulted in a list of twenty-six codes and agreed-upon definitions. After this step, one coder independently coded all the messages individually. The two coders then collectively reviewed any new coding categories that emerged during this process and finalized the definitions. This iterative process led to a code book with thirty-six codes and their descriptions. These codes are listed in Table 3. For answering research question 3 , we reviewed the first message of each thread and developed categories for the types of support that women are seeking on the forums. For answering research question 4 , we analyzed all the messages that were either offering support or were providing information to support women in OSS.

\section{Results}

In this section, we present the results for each of the research questions. First, we present results for the individual forums and then the cumulative results for the five forums to demonstrate patterns and trends in these forums.

\subsection{Activity Level on the Forums}

Data collection from five OSS discussion forum archives resulted in the collection of 10,698 messages. Table 1 presents the total number of messages from each forum and the date range of forum activity. The age of the forums ranges from thirteen to seventeen years. The data collected for GNOME spans twelve years and two months. For the KDE mailing list, the dataset includes fifteen years and eight months of discussions. The FEDORA dataset spans thirteen years and four months. The Ubuntu archives contain messages that range twelve years and eleven months. Finally, the Debian archives include messages written over fourteen years and six months. The number of messages posted in each forum varies significantly. On one end, the Fedora archives contain only 169 messages, while, on the other end, the Debian archives contain 5,247 messages.

Table 1 Number of Post and Age of the forums

\begin{tabular}{|l|l|l|}
\hline OSS & No. of posts & Date Range \\
\hline Debian & 5,247 & June '04 to Dec '18 \\
\hline FEDORA & 169 & Jun '06 to Sep '18 \\
\hline GNOME & 418 & July '05 to Oct '18 \\
\hline KDE & 422 & Feb '02 to Oct '17 \\
\hline UBUNTU & 4,442 & Feb '06 to Jan '19 \\
\hline Total & $\mathbf{1 0 , 6 9 8}$ & \\
\hline
\end{tabular}

The number of participants on each forum ranged from fifty-seven in Fedora to 587 in Debian. The total number of participants across the forums is 1 , 344. In our dataset, we did not check to see if there were 1 , 344 unique participants or some of these were participating in multiple forums. Within each forum, the total is the number of unique participants. If we encountered multiple emails for the same participant, we considered it as one participant.

Table 2 Number of participants on each forum

\begin{tabular}{|l|l|}
\hline Forum & Number of Participants \\
\hline Debian & 587 \\
\hline Fedora & 57 \\
\hline Gnome & 120 \\
\hline KDE & 121 \\
\hline Ubuntu & 459 \\
\hline Total & 1,344 \\
\hline
\end{tabular}

In twelve years and three months, there were 169 messages posted on the Fedora mailing list. The range in number of messages posted per month was from zero to twenty-five. Generally, there were five or fewer messages posted per month. In the 145 months, there were only eight months when more than five messages were posted. In the next section, we further examine those months with more than five messages. The average number of messages per month was at least one.

In fifteen years and eight months, there were 422 messages posted on the KDE mailing list. The range of messages posted per month was from zero to twentyeight. On average, there were at least two messages posted per month. 
In thirteen years and four months, 418 messages were posted on the GNOME mailing list. The range of messages posted per month was from zero to fortyfive. Mostly, five or fewer messages were posted per month on this mailing list. More than five messages were posted in twenty-eight months. On average, there were at least two messages posted per month.

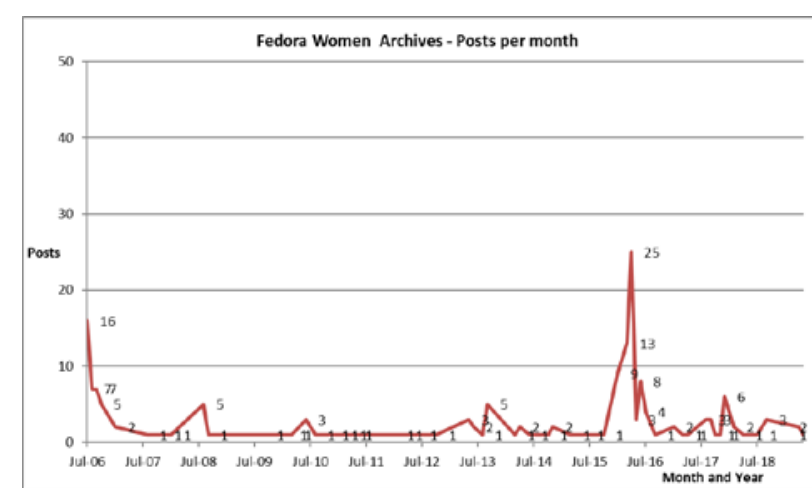

Figure 1 Fedora archives posts per month

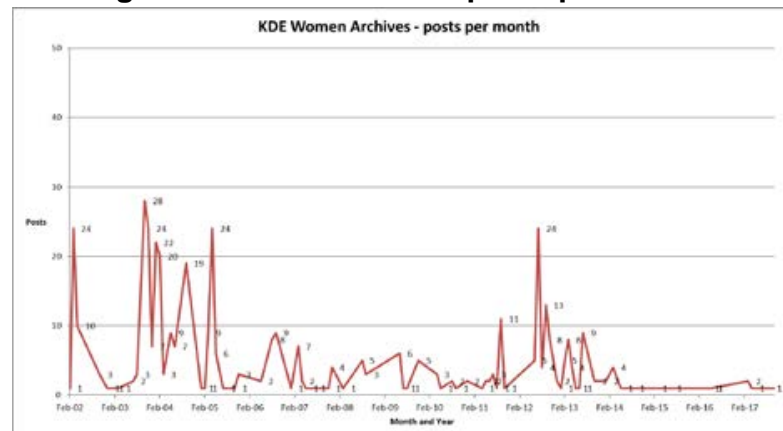

Figure 2 KDE Archives Posts per month

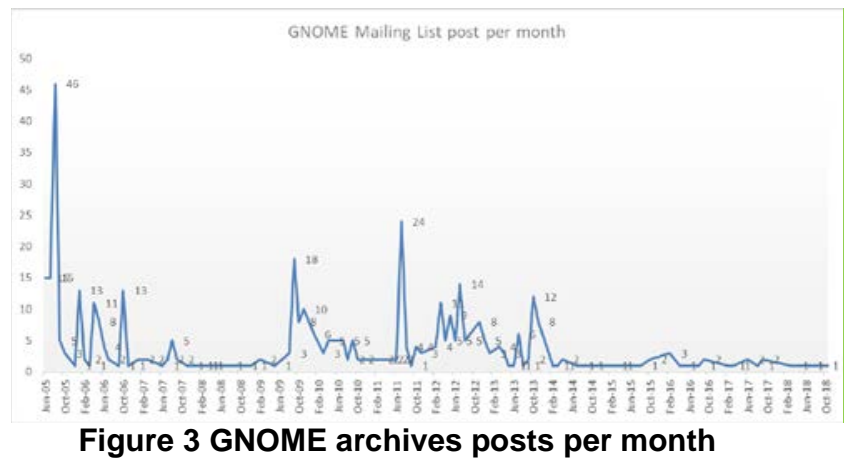

In thirteen years, there were 459 messages posted on the Ubuntu mailing list. The range of messages posted per month was from zero to 187. (The graph below cuts off at 140 to give a better representation of the activity level. The cutoff month was March 2006 with 187 messages.) On average, at least twenty-eight messages were posted per month.

The Debian women mailing list spanned fifteen years and included 587 posted messages. (The graph below cuts off at 200 messages to give a better representation of the activity level. The months that are not visible in the graph below are August 2004 [352], July 2004 [325], June 2005 [308], May 2005 [235], April 2005 [214] and Aug 2005 ([04].) This list is the most active in our dataset, and the highest number of messages in one month was 352 messages. On average, at least thirty messages were posted per month.

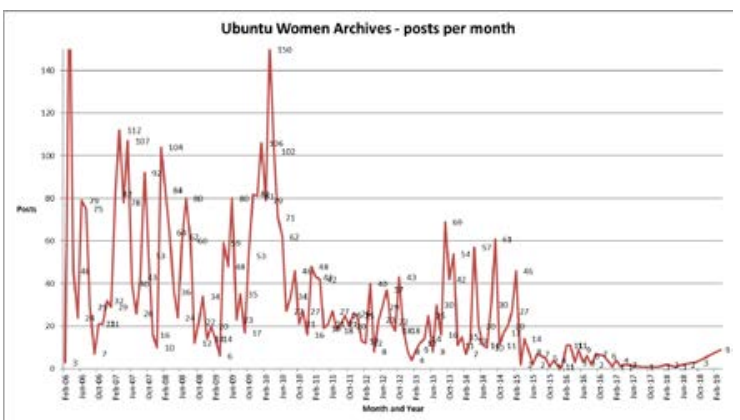

Figure 4 Ubuntu Archives Posts per month

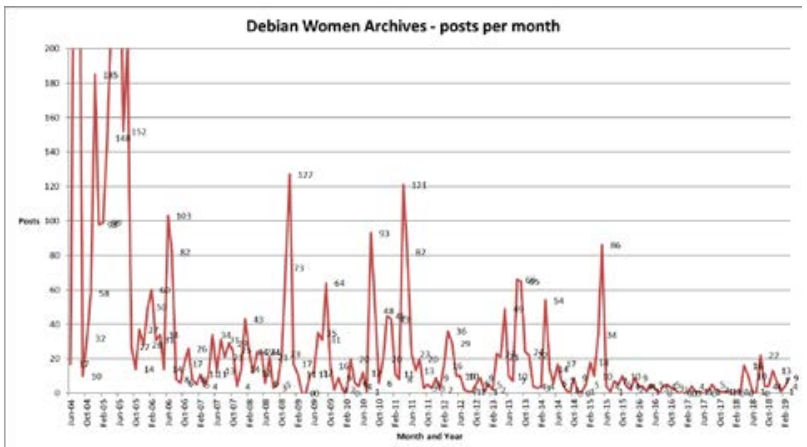

Figure 5 Debian Archives Posts per month

After all the messages were coded, several common themes that showcased the role of these forums emerged. The forums were places to share experiences, to create communities, to discuss the creation of communities, to seek help in technical matters, to encourage participation, and to highlight opportunities for collaboration, networking and mentoring.

4.2.1 Most common topics of posts: For identifying the most common topics in each forum, we used the codes described above. We coded the occurrence of each code category to discover the most frequent topics and the specific details are presented in Table 3. In the following section, we present the top five categories of messages for each forum.

In the Debian archives, which spanned fourteen years, we found that the most common message category was women-focused events, announcements, and discussions. There were 886 messages in this category out of 5,247 messages in the archive, making this category $16 \%$ of the total. 674 of the messages, or thirteen percent of the total, concerned technology 
questions or issues regarding the forum itself (or another women's forum) 509 messages, of $10 \%$ of the total, dealt with "sexism" in some form. Posts related to technology issues, access problems, troubleshooting, and learning new technologies made up about 9\% (482 messages), while 333 messages involved "introductions." This category includes both posts in which someone is introducing herself and posts in which others are welcoming new members to the forum.

In the Gnome archives, which spanned fourteen years, there were 418 messages. Women-focused events made up the largest category of messages with a total of 117 , or $28 \%$. Messages about general events, promotion related to women-focused groups or event introductions of new members, and membership questions formed for about seven percent each

Table 3 - Number of Messages by Topic

\begin{tabular}{|c|c|c|c|c|c|}
\hline Code Category & $\mathrm{D}^{*}$ & $\mathrm{G}^{*}$ & $\mathrm{~K}^{*}$ & $\mathrm{~F}^{*}$ & U* \\
\hline $\begin{array}{l}\text { Announcement - } \\
\text { General }\end{array}$ & 16 & & & & 9 \\
\hline Award - Women & 60 & & & 2 & 123 \\
\hline CFP & 67 & 19 & 16 & 8 & 50 \\
\hline Code of Conduct & 41 & 7 & & 2 & 69 \\
\hline Discrimination & 136 & & & & 10 \\
\hline ELT & 33 & & & & 1 \\
\hline Event - General & 121 & 32 & 9 & 2 & 128 \\
\hline Event - WF & 886 & 115 & 114 & 42 & 645 \\
\hline Feedback & 13 & 3 & & & 38 \\
\hline Feminism & 4 & & 6 & & 3 \\
\hline Greetings & 19 & & 4 & & 10 \\
\hline $\begin{array}{l}\text { Greetings, General } \\
\text { Question, ELT, } \\
\text { Award, Other WFs }\end{array}$ & & $\begin{array}{l}1 \\
\text { each }\end{array}$ & & $\begin{array}{l}1 \\
\text { eac } \\
h\end{array}$ & \\
\hline Introduction & 333 & 29 & 83 & 19 & 753 \\
\hline Job Announcement & 35 & 25 & 12 & 11 & 24 \\
\hline Meeting - WF & 124 & 8 & 14 & 2 & 424 \\
\hline Membership & 251 & 24 & 22 & & 268 \\
\hline News - WF & 165 & 20 & 14 & 14 & 183 \\
\hline News - General & & & & & 13 \\
\hline News - OSS & & & & & 8 \\
\hline OSS Culture & 104 & & & & 20 \\
\hline Other & 32 & & & & 32 \\
\hline Promotion - WF & 96 & 31 & & 12 & 11 \\
\hline Purpose/Goals & 213 & & 5 & 12 & 232 \\
\hline Research - General & 1 & 2 & & & \\
\hline Research - WF & 96 & 5 & 4 & 2 & 20 \\
\hline Sexism & 509 & 0 & & & 223 \\
\hline Stories & 25 & & & & 48 \\
\hline Survey Request & 154 & & 19 & 7 & 64 \\
\hline Tech - General & 17 & 4 & 2 & 3 & 39 \\
\hline Tech - WF & 674 & 6 & 50 & 9 & 501 \\
\hline Tech Issue & 482 & 11 & 21 & 14 & 145 \\
\hline Translation & 281 & 26 & 21 & & 72 \\
\hline Women in Technology & 259 & 21 & 2 & & 132 \\
\hline
\end{tabular}

*D=Debian, G=GNOME, K=KDE, F= Fedora, U=Ubuntu

In the KDE archives, which spanned fifteen years and had 423 messages, women-focused events, at 114 messages, constituted about $27 \%$ of the total. Eightythree messages involved "introductions," making this category about $20 \%$ of the total. Fifty messages, or $11 \%$ of the total, concerned technology questions or issues regarding the forum itself (or another women's forum). Forum membership issues and technology issues each had a total of twenty-two messages, 5.2 percent of the total.

In the Fedora archives, which spanned twelve years and included 173 messages, posts related to womenfocused events or activities (forty-two in number) constituted almost a quarter of total. Twenty-two "introduction" messages were posted, making up about $13 \%$ of the total. Three other categories-womenfocused news stories, promotion for women's groups and events, and technology questions and issues-had fourteen messages each, or about eight percent of the total.

In the Ubuntu archives, which spanned twelve years and included 4,442 messages, the largest number of messages concerned "introductions." These 753 posts accounted for almost $17 \%$ of the total. Posts related to women-focused activities or events made up about $45.5 \%$ (645 messages). Technology questions or issues related to the forum itself (or another women's forum) were the subject of 501 messages, or $11 \%$ of the total. Finally, the 424 posts concerning group meetings, minutes, etc. constituted $10 \%$ of the total, while the 268 membership-related constituted 6\%.

Across the five forums, we found that messages related to women-focused events or activities were very frequent. This category includes posts related to mentoring. Other common topics across the forums include introductions, community membership and technology-related help-seeking either about womencentered forums or about technology in general. Translation requests from non-English speaking members or offers to translate were also common. Across the forums, discussion about sexism, discrimination, feminism, and women in technology was common but not the central focus in any of them.

4.2.2 Topics that generated the most discussion: Next, we were interested in the topics that generated the most discussion in these forums. For this analysis, we coded all the messages for the month with the largest number of posts in each forum. Table 8 presents the summary of the most active months on each forum. It includes the number of messages and topics discussed in that month.

In the Debian archives, the most active month was August 2004 with 352 messages. The top five categories were sexism (eighty-five messages), 
technology questions related to women's forums (seventy-six messages), OSS culture (forty-two messages) and discrimination (forty-five messages).

In the Fedora archives, the most active month was April 2016 with twenty-five messages. Thirteen messages concerned women-focused events or activities, and twelve messages discussed promoting women's groups and activities.

In the Gnome archives, the most active month was August 2005 with forty-six messages, out of which seventeen are about translation, twelve are general discussions about women in technology, and eleven messages are about women-focused events and activities.

In the KDE archives, the most active month was October 2003. Of the twenty-eight messages for this month, thirteen concern women-focused events or activities. Twelve messages, in turn, concern translation, while three deal with technology issues.

Table 3 - Summary of the most active months

\begin{tabular}{|l|l|}
\hline Forum & $\begin{array}{l}\text { Month, Number of messages and topics } \\
\text { discussed }\end{array}$ \\
\hline Debian & $\begin{array}{l}\text { August 2004 - (352 messages), Tech - WF, } \\
\text { Membership, Sexism, Tech Issue, Stories, } \\
\text { Translation, Research - WF, News - WF, } \\
\text { OSS Culture, Discrimination, Women in } \\
\text { Technology, Introduction, CFP, Survey } \\
\text { Request, Event - General, Announcement - } \\
\text { General }\end{array}$ \\
\hline Fedora & $\begin{array}{l}\text { April 2016 - (25 messages) Women- } \\
\text { Focused Event, Women-Focused Promotion }\end{array}$ \\
\hline Gnome & $\begin{array}{l}\text { Aug 2005 (46 messages) Promotion - } \\
\text { Women-Focused, Women in Technology, } \\
\text { Translations, Job Announcement, General } \\
\text { Event, Introduction, Greetings }\end{array}$ \\
\hline KDE & $\begin{array}{l}\text { Oct 2003 (28 messages) KDE Women } \\
\text { Meeting, Volunteers for the event, call for } \\
\text { translators and tech issues }\end{array}$ \\
\hline Ubuntu & $\begin{array}{l}\text { March 2006 - (187 messages), Introduction, } \\
\text { Membership, Event - WF, OSS Culture, } \\
\text { Tech - WF, Translation, Greetings, Code of } \\
\text { Conduct, Tech Issues, Event - General, } \\
\text { Purpose/Goals, Tech - WF }\end{array}$ \\
\hline
\end{tabular}

In the Ubuntu archives, the most active month was March 2006 with 187 messages. Of this total, ninetyfive messages were introductions, while thirty-six dealt with technology-related questions related to women's forums. Additionally, twenty-one messages concerned translation, and seventeen concerned membership issues. OSS culture and codes of conduct were also discussed in this month. Across the forums, we found that messages about women-focused activities or events and messages about translations formed the most active discussion topics. Moreover, in Debian, we found that sexism, OSS culture, and discrimination were discussed in the month with the highest activity. The Gnome archives also had an active discussion about women's issues in technology in its most active month. Finally, Ubuntu's most active month also included discussions of OSS culture and codes of conduct.

\subsection{Types of support being sought}

As described in the earlier sections, these online forums serve a variety of purposes, including providing spaces for announcements, networking, and support (technical, social and professional). In this section, we focus on the requests for help or social support posted by the participants in these forums. For a more indepth look, we focused only on the messages that were requests for help. We then categorized the help-seeking messages into broad categories via qualitative analysis. For each coded category, we provide here the code name, description and representative excerpts from the messages on the forums.

Technical help: In this type of help message, a forum participant has become stuck while trying either to figure out how to use new software or to learn a new skillset, and asks for assistance. There are also help requests concerning technical issues identified in the software.

Help related to managing the women-focused forum's technology: This category includes list moderation, website development, logo development, wiki maintenance, etc. In the following example, someone who is stepping down as a moderator of the list seeks volunteers to take over and contribute to the management of the women listserv.

"Hello All,

This mail has been on my to-do list for a long time so here goes. Since I will be busy with gsoc and other floss development work, I

would like to reduce the lists I manage, and hand over admin privs of this list to any individual(s) interested in taking over the work of discarding spam messages --not a very hard task to learn so its a nice place to get started if you have a few minutes per day/week......"

Getting started with development or volunteer work on a to-do list for a project: In these posts, women discuss their desire to get started with a project. They volunteer their skills, seek help to get started and learn the process of contributing to OSS.

"Hi!

I'm (A-Volunteer), . It would be great 
if anyone could help me in getting started (like what do I start with? Any small bugs that I could fix or small piece of code $i$ could work on??) I also want to know if I could propose any new feature...."

Translation help: This type of help involves the translation of content into another language. Volunteers help women in other countries to access content. Many examples of this type of help were seen across the forums. Languages included Spanish, Portuguese, Filipino, French, Vietnamese, Hungarian, etc. In the excerpt below, a participant volunteers to help out and attempts to recruit more volunteers for the task of translation.

"Because I'm a Frenchie interested in doing some translation work and because I believe Debian-Women is Good, I hereby announce that it would be my pleasure to translate (part of?) the website to French. Unfortunately it's not so easy for me to find both time and energy to do such a big amount of work these days (hence the "part of" up there) but I hope in time I can get there. Any help would be very welcome of course."

Job search help: In these posts, participants are looking for job opportunities for their particular skill sets and also seeking help about the process of the job search in the OSS context. So here we have questions like, "How do I get a job working in Linux?”

Peer review: In these posts, participants seek help from fellow forum members about something that they have completed, such as a software code or documentation or presentation tips for a talk, etc. They ask for feedback on their work from their peers.

Advice: In these messages, participants seek guidance from other forum members about a variety of issues, mostly the future of the mailing list or forum, the presence of women in OSS communities, social media presence, etc. These posts also include advice for hosting future events and best practices for management. In some instances, women seek advice concerning harassment and discriminating behavior in OSS culture.

Event management help: These requests are very common. Most often, volunteers are sought to help with hosting events. Some specific examples are: Help host a bug day (Gnome), Ada Lovelace day (KDE), FOSS seminar, hackathons, Grace Hopper events, etc. This category also includes requests for membership to various committees and events.

Mentors: This category includes requests for mentorship and guidance. Some of these requests are specific, while some were more general in their approach. This category also includes discussions of mentoring events hosted for women and other similar opportunities for becoming a mentor or mentee.

Research / survey help: All the forums had discussions about OSS culture as well as messages from people conducting research on the presence of women in OSS. Survey requests from academia and the corporate sector were common.

The types of help that women are seeking range from technical issues to requests for mentoring or collaboration to requests for participation in initiatives. Requests for translations were also common across all five forums.

\subsection{Types of received social support}

To get a better understanding of the types of social support that women receive on these forums, we analyzed the messages on the forums, with a focus on the responses to posts. This analysis included all messages, and coded the elements of each message that offered social support to the participant who initiated the thread. The received social support elements did not depend on the help being sought but on the help being received. These messages also included unsolicited offers of help and shared opportunities.

The following qualitative themes emerged from this coding. Relevant excerpts from the messages are presented for some of the themes. (Due to space constraints, an excerpt for each theme is not presented.)

Networking opportunities: This category was the most common, as reported in the previous section. It includes messages that announce networking opportunities such as invitations for talks, lectures, tutorials, job opportunities, event invites, outreach programs for women, social events for women, mentoring opportunities, mentoring for specific events such as outreach, GHC, etc.

Awards: These forums included many messages announcing women-focused awards or accomplishments across OSS projects and communities. They were not necessarily focused on the community in which they were posted but were rather general awards for women in OSS or women in technology. These posts are considered support messages, as they help women receive recognition. Additionally, they demonstrate the respective community's interest in diversity and inclusion, and open doors for women. This type of recognition for women is encouraging for both the women who are recognized and the other women who see successful role models in OSS.

Support group: This element was a key component of the responses posted in these forums. Even if 
responses did not directly answer a question, they provided support for the original poster. The responding member would point her in the right direction, or, as often was the case, she would share her own relevant experiences. Experiences concerning successful learning, time management, project contribution, dealing with technical and social challenges, etc. were all discussed in a large number of messages, creating a supportive overall environment. Even if the forums were not always active, they still served as places to go to post questions and receive support. The women participating in the forums could count on the support from this community.

Mentorship: Many veteran contributors, male and female, offered to mentor newcomers into a project and shared best practices and guidelines for contributing to OSS. They would post an initial message as a volunteer or would pick up a request for help and start helping the original poster with technical issues, even if they were not formally mentoring the person. Formal and informal mentorship were both observed.

These forums served as venues for discussion and brainstorming, especially concerning issues that women encounter in OSS. We did not observe any preference for what that issue might be. While frequent, technical help was not the most common activity. Women did seek and receive technical help, and shared technical resources with each other, but the overall majority of their forum use involved seeking and providing different kinds of social support. Whether technical or social, other women were equally supportive and available to share, discuss, and brainstorm ideas with the original poster.

These forums also played an important role in providing women with the space to discuss women's issues. We observed general discussions about the status of women in technology and women in OSS, often sharing the latest research, articles, and media reports. Similarly, we also observed discussions about discrimination, sexism, and feminism. Even though these were not the most common discussions, they often could not be conducted in the main project/software mailing lists. By facilitating such discussions and providing support relevant to the associated issues, these forums served the role of safe spaces for their participants.

\section{Discussion and Conclusion}

The results demonstrate that the value of these online forums lies in their role as safe spaces providing social support to the minorities who participate in these forums, and not merely as technical support forums. This result is surprising because the assumption has been that these forums are for technical support and are about a particular software. It can be argued that positive interactions experienced on these forums would empower women, embolden them to champion other minorities, and have much more far reaching impact on the overall presence of marginalized voices in Open Source Software. The impact would intuitively go beyond the particular software where the discussion is happening.

In past research, [7] studied women's participation in Stack Overflow to understand the influence of Peer Parity (defined as when an individual can identify at least one other peer interacting in a community). The authors discussed how showcasing the success of women, pairing women for guidance and revealing user identities will all positively influence peer parity and provide encouragement for women to participate in OSS. When we look at the results of the present project from the "peer parity" perspective, we find that the role of the forum is to allow women (as an inclusive term) to support one another and not to be a technical help venue. Importantly, the topics that generated the most discussion across all the forums were women-focused events and activities, showing that active networking happens on these forums. We found messages that celebrated the successes of women as well as messages that paired women in mentor-mentee relationships. The majority of women participants on these forums used their names and affiliations in their signatures, demonstrating that they were comfortable revealing their identities. All these findings point towards the value of these women-focused spaces in OSS communities.

Based on this work and other similar research referenced in this article, we urge OSS communities to support the creation and growth of such safe spaces for women, not only to support their own software but to respond to the overall under representation of women and minorities in the OSS environment. This in turn will benefit innovation and the economy for everyone, and will achieve greater equity for marginalized groups.

In future work, we will continue to focus on a deeper understanding of these spaces, individual participants, their journeys and their perspectives on the value and impact of these spaces through in-depth interviews. In the next phase of analysis, we are specifically focusing on the threads that concern OSS culture, discrimination, sexism and the status of women in IT. These issues will help us to understand these online communities through the notion of “community smell." Community smells reflect suboptimal organizational and socio-technical patterns in the organizational structures of software communities [10] 
In conclusion, we found that these forums are support-based networking spaces for women rather than technical problem-solving forums. In these spaces, women receive and provide social support, helping each other to succeed in the hostile OSS environment by sharing experiences, expertise and opportunities. Overall, these forums provide safe spaces in which to interact that foster gender diversity and lead to greater inclusivity instead of creating barriers for women who participate.

\section{References}

[1] A. Adam, . D. Howcroft, and H. Richardson.(2004) “A Decade of Neglect: Reflecting on Gender and IS,” New Technology, Work and Employment Volume 19, Number 3, 2004, pp. 222-240

[2] Amy S., Fiesler, Casey, \& Bruckman, Amy S. (2016). An archive of their own. $34^{\text {th }}$ Annural CHI Conference on Human Factors in Computing Systems, 2574-2585.

[3] C Mendez A Sarma, and M Burnett . (2018). Open Source Barriers to Entry, Revisited: A Tools Perspective. Proceedings of ACM ICSE Conference, 2018, 1-12. https://doi.org/10.475/123_4

[4] C. Dunkel-Schetter, \& L.A. Skokan. (1990). Determinants of social support provision in personal relationships. Journal of Social and Personal Relationships, 7(4), 437-450.

[5] C.B. Hanhardt. Safe space: Gay neighborhood history and the politics of violence. Duke University Press, 2013.

[6] C.E. Cutrona. \& D.W. Russell. (1990). Type of social support and specific stress: Toward a theory of optimal matching. In B. R. Sarason, I. G. Sarason, \& G. R. Pierce (Eds.), Wiley series on personality processes. Social support: An interactional view (pp. 319-366). Oxford, England: John Wiley \& Sons.

[7] D Ford, A Harkins, and C Parnin (2017). Someone Like Me: How Does Peer Parity Influence Participation of Women on Stack Overflow? 2017 IEEE Symposium on Visual Languages and Human-Centric Computing (VL/HCC), 239-243. DOI: 10.1109/VLHCC.2017.8103473

[8] D. Nafus (2012). 'Patches Don't Have Gender': What Is Not Open in Open Source Software. New Media \& Society, 14(4), 669-683. 10.1177/1461444811422887

[9] D. Rock and H. Grant. (2016). Why diverse teams are smarter? Harvard Business Review, November 2016 retrieved on 5/15/2019 from https://hbr.org/2016/11/why-diverse-teams-are-smarter

[10] F. Palomba, D. A. Tamburri, A. Serebrenik, A. Zaidman, F. A. Fontana, and R. Oliveto. 2018. How do community smells influence code smells? In Proceedings of the 40th International Conference on Software Engineering: Companion Proceeedings (ICSE '18). ACM, New York, NY, USA, 240-241.

[11] J. Holt-Lunstad. and B.N. Uchino. (2015) Social support and health, chapter 10 in In Glanz, K., In Rimer, B. K.,
\& In Viswanath, K. (2015). Health behavior: Theory, research, and practice. San Francisco, CA : JosseyBass, a Wiley Brand.

[12] K. Crenshaw. (1991) Mapping the margins: Intersectionality, identity politics, and violence against women of color, Stanford Law Review, 43(6): 1241-99.

[13] L. A. Von Hellens, and S. Nielsen.(2001) “Australian Women in IT." Communications of the ACM, Volume 44, Number 7, 2001, pp. 46-52.

[14] M. K. Scheuerman,, S.M. Branham, \& F. Hamidi F. (2018). Safe spaces and safe places: Unpacking technology-mediated experiences of safety and harm with transgender people. Proceedings of the ACM on Human-Computer Interaction, 2(CSCW), 155.

[15] M. Rowe, R. Lewis, \& C. Wiper. (2018). Misogyny Online: Extending the boundaries of Hate Crime. Journal of Gender-Based Violence, 2(3), 519536. https://doi.org/10.1332/239868018X153753044726 35

[16] M.G.J. Cohoon.. 2011. Which Gender Differences Matter for High-Tech Entrepreneurship?. Open Source Business Resource, (July 2011). http://timreview.ca/article/454

[17] NCWIT National Council of Women in Information Technology https://www.ncwit.org/sites/default/files/resources/btn_0 3092016_web.pdf.(2016

[18] Paul Dourish and Genevieve Bell. 2007. The Infrastructure of Experience and the Experience of Infrastructure: Meaning and Structure in Everyday Encounters with Space. Environment and Planning B: Planning and Design 34, 3: 414-430. https://doi.org/10.1068/b32035t

[19] R Lewis, E Sharp, J Remnant, and R Redpath (2015). 'Safe Spaces': Experiences of Feminist Women-Only Space. Sociological Research Online, 20(4), 1-14.

[20] R. Lewis, M. Rowe \& C. Wiper. (2017). Online abuse of feminists as an emerging form of violence against women and girls. British Journal of Criminology, 57(6), 1462-1481. https://doi.org/10.1093/bjc/azw073

[21] R. Pain. 1991. Space, sexual violence and social control: integrating geographical and feminist analyses of women's fear of crime. Progress in Human Geography, 15 p. 415-431. [doi:10.1177/030913259101500403]

[22] S. Cobb. (1976). Social support as a moderator of life stress. Psychosomatic Medicine, 38(5), 300-314.

[23] S. Herring, K. Job-Sluder, R. Scheckler, and S. Barab. "Searching for safety online: Managing" trolling" in a feminist forum." The information society 18, no. 5 (2002): 371-384.

[24] V. Singh and W. Brandon (2019) Open Source Software Community Inclusion Initiatives to Support Women Participation. In: Bordeleau F., Sillitti A., Meirelles P., Lenarduzzi V. (eds) Open Source Systems. OSS 2019. IFIP Advances in Information and Communication Technology, vol 556. Springer, Cham

[25] V. Singh. (2019). "Investigation into the Women Only Spaces in Open Source Software communities" Second Workshop on Gender Equality (GE@ICSE) at International Conference on Software Engineering (ICSE), Montreal, Canada, May 27th, 2019. 